\title{
The benefit of enhanced contractility in the infarct borderzone: a virtual experiment
}

\author{
Zhihong Zhang 1,2, Kay Sun 1,2, David A. Saloner ${ }^{2,3}$, Arthur W. Wallace ${ }^{2,4}$, Liang Ge ${ }^{1,2,5}$, Anthony J. Baker ${ }^{2,6}$, \\ Julius M. Guccione ${ }^{1,2,5}$ and Mark B. Ratcliffe ${ }^{1,2,5 *}$
}

1 Department of Surgery, University of California San Francisco, San Francisco, CA, USA

${ }^{2}$ San Francisco Veterans Affairs Medical Center, San Francisco, CA

${ }^{3}$ Department of Radiology, University of California San Francisco, San Francisco, CA, USA

${ }^{4}$ Department of Anesthesia, University of California San Francisco, San Francisco, CA, USA

${ }^{5}$ Department of Bioengineering, University of California San Francisco, San Francisco, CA, USA

${ }^{6}$ Department of Medicine, University of California San Francisco, San Francisco, CA, USA

\section{Edited by:}

Guillermo A. Cecchi, IBM Watson

Research Center, USA

Reviewed by:

Flavio H. Fenton, Cornell University,

USA

Viatcheslav Gurev, International

Business Machines, USA

*Correspondence:

Mark B. Ratcliffe, Surgical Service

(112), San Francisco Veterans Affairs

Medical Center, 4150 Clement Street,

San Francisco, CA 94121, USA.

e-mail:mark.ratcliffe@va.gov

\begin{abstract}
Objectives: Contractile function in the normally perfused infarct borderzone (BZ) is depressed. However, the impact of reduced BZ contractility on left ventricular (LV) pump function is unknown. As a consequence, there have been no therapies specifically designed to improve BZ contractility. We tested the hypothesis that an improvement in borderzone contractility will improve LV pump function. Methods: From a previously reported study, magnetic resonance imaging (MRI) images with non-invasive tags were used to calculate 3D myocardial strain in five sheep 16 weeks after anteroapical myocardial infarction. Animalspecific finite element (FE) models were created using MRI data and LV pressure obtained at early diastolic filling. Analysis of borderzone function using those FE models has been previously reported. Chamber stiffness, pump function (Starling's law) and stress in the fiber, cross fiber, and circumferential directions were calculated. Animal-specific FE models were performed for three cases: (a) impaired BZ contractility (INJURED); (b) BZ-contractility fully restored (100\% BZ IMPROVEMENT); or (c) BZ-contractility partially restored (50\% BZ IMPROVEMENT). Results: 100\% BZ IMPROVEMENT and 50\% BZ IMPROVEMENT both caused an upward shift in the Starling relationship, resulting in a large (36 and 26\%) increase in stroke volume at $L V P_{E D}=20 \mathrm{mmHg}(8.0 \mathrm{ml}, p<0.001)$. Moreover, there were a leftward shift in the end-systolic pressure volume relationship, resulting in a 7 and $5 \%$ increase in LVP $P_{\text {ES }}$ at $110 \mathrm{mmHg}(7.7 \mathrm{ml}, p<0.005)$. It showed that even 50\% BZ IMPROVEMENT was sufficient to drive much of the calculated increase in function. Conclusion: Improved borderzone contractility has a beneficial effect on LV pump function. Partial improvement of borderzone contractility was sufficient to drive much of the calculated increase in function. Therapies specifically designed to improve borderzone contractility should be developed.
\end{abstract}

Keywords: myocardial Infarction, borderzone, finite element

\section{INTRODUCTION}

It has been known since the mid 1980s that systolic shortening and wall thickening are depressed in the non-ischemic infarct borderzone (Homans et al., 1985). More recently, Jackson et al. (2002) described infarct extension after anteroapical myocardial infarction in sheep, which surprisingly, occurs in the face of normal borderzone (BZ) blood flow.

It was initially thought that reduced borderzone shortening was due to mechanical tethering (high systolic stress) by the infarct (Kramer et al., 1996). To better clarify the cause, we previously created a finite element (FE) model of the left ventricle (LV) that was based on magnetic resonance imaging (MRI) data obtained after anteroapical MI in sheep (Guccione et al., 2001; Walker et al., 2005). Model output was optimized by comparing strain predicted by the model with strain measured using MRI. Surprisingly, the FE simulations showed that BZ contractility was significantly depressed by
$50 \%$ compared to the remote uninfarcted myocardium (Guccione et al., 2001). It is therefore logical to consider BZcontractility improvement as a potential therapy for LV dysfunction after MI.

In part because the cause of depressed function in the normally perfused infarct borderzone is unclear, there have been no therapies specifically designed to improve borderzone contractility. We therefore tested the hypothesis that an improvement in borderzone contractility will improve LV pump function.

\section{MATERIALS AND METHODS FINITE ELEMENT MODEL}

This work used previously reported five animal-specific FE models of ovine LV with an anteroapical infarct (Walker et al., 2005; Sun et al., 2009). The LV wall in each FE model was divided into four regions: remote myocardium with normal passive myocardium properties, the borderzone which was broken 
into two regions near and far from the infarct with normal passive myocardium properties, but reduced active contraction and the dyskinetic infarct with increased stiffness and no active contraction (Figure 1). Each FE model has three transmural layers and has 2496 elements. Note that the two BZs have different myofiber contractilities but identical passive wall stiffness. The previously determined regional in vivo myofiber contractilities were used in this work as the baseline case (Sun et al., 2010). The parameters (passive stiffness $C$ and active contraction $T_{\max }$ ) used in this study are listed in Tables 1 and 2 .

In order to investigate the impact of BZ-contractility improvement on LV pump function, we numerically increased the BZ contractility, $T_{\max }$, as follows:

$$
\left.T_{\max }^{\mathrm{BZ}}\right|_{\text {Ehd }}=T_{\max }^{\mathrm{BZ}}+P \% \times\left(T_{\max }^{\mathrm{RE}}-T_{\max }^{\mathrm{BZ}}\right)
$$

where $T_{\max }^{\mathrm{BZ}}$ and $T_{\max }^{\mathrm{RE}}$ are the baseline contractilities of the BZs and the Remote region, $\left.T_{\max }^{\mathrm{BZ}}\right|_{\text {Ehd }}$ is the new BZ contractility after virtual contractility enhancement, and is the percentage of enhancement. We investigated four different levels of enhancement, namely $25,50,75$, and $100 \%$. The baseline case would be named as the INJURED and the virtual enhancement cases would be named as $P \%$ BZ IMPROVEMENT. The pressure loading conditions for the BASELINE models were experimentally determined and the BZ IMPROVEMENT cases were kept the same as the BASELINE case.

\section{DATA ANALYSIS}

\section{Calculation of end-systolic pressure-volume relationships}

End-systolic solutions were obtained at ranges of end-diastolic (from 0 to $17 \mathrm{mmHg}$ ) and end-systolic (from 10 to $90 \mathrm{mmHg}$ ) chamber pressures. The LVP $\mathrm{ES}_{\text {S }}$ and $\mathrm{LVV}_{\mathrm{ES}}$, determined from the FE model were fit to a linear equation by means of least square regression analysis (Kleinbaum et al., 1988),

$\mathrm{LVP}_{\mathrm{ES}}=E_{\mathrm{ES}}\left(\mathrm{LVV}_{\mathrm{ES}}-V_{0}\right)$

where $V_{O}$ is the volume intercept and $E_{\mathrm{ES}}$ is the slope of the $\mathrm{LV}$ elastance.

The diastolic pressure volume relationship was assumed to be unchanged.

\section{Calculation of stroke volume/LVP $P_{E D}$ (Starling) relationship}

For each level of passive constraint, the stroke volume (SV) versus the $\mathrm{LVP}_{\mathrm{ED}}$ (Starling) relationship was calculated from diastolic and systolic pressure-volume regression. SV for each level of preload

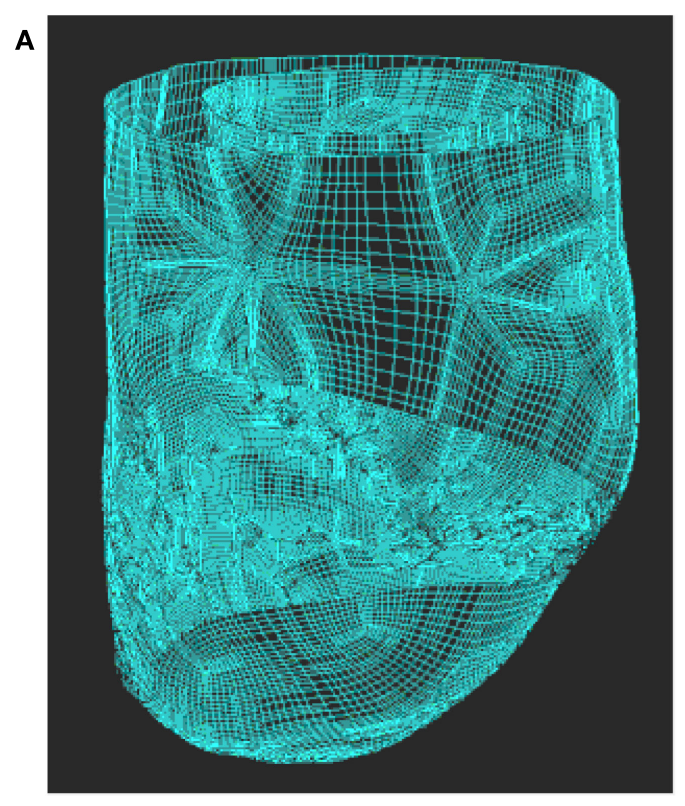

B

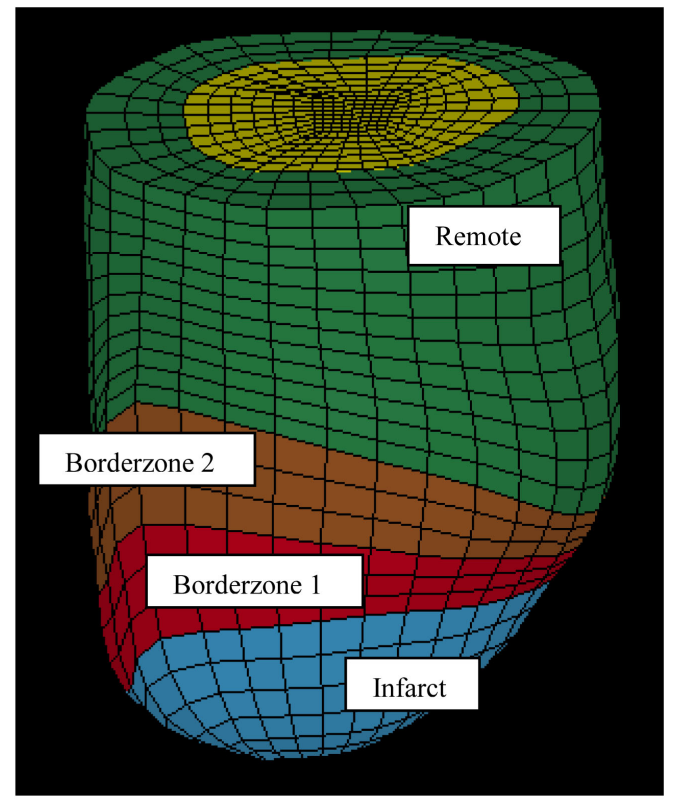

FIGURE 1 | Finite element model of the LV with anteroapical MI. Animal-specific contours were generated from MRI (A). Solid mesh (B) was broken into four regions (green = remote, brown and red = borderzone, and blue $=$ infarct).

Table $1 \mid T_{\max }$ values used for the baseline case and the borderzone contractility enhancement cases.

\begin{tabular}{lcllll}
\hline & Baseline & 25\% BZ improvement & 50\% BZ improvement & 75\% BZ improvement & 100\% BZ improvement \\
\hline Remote $(\mathrm{kPa})$ & $434.9 \pm 413$ & $434.9 \pm 413$ & $434.9 \pm 413$ & $434.9 \pm 413$ & $434.9 \pm 413$ \\
Borderzone 2 $(\mathrm{kPa})$ & $95.9 \pm 36$ & $180.6 \pm 127.1$ & $265.4 \pm 222$ & $350.2 \pm 317$ & $434.9 \pm 413$ \\
Borderzone 1 $(\mathrm{kPa})$ & $59.3 \pm 41$ & $153.2 \pm 122.7$ & $247.1 \pm 218$ & $341.0 \pm 315$ & $434.9 \pm 413$
\end{tabular}


Table 2 | Passive material property $(C)$ values used for all cases.

\begin{tabular}{lc}
\hline & $\boldsymbol{C}(\mathbf{k P a})$ \\
\hline Remote & $0.946 \pm 0.35$ \\
Borderzone 1 & $0.946 \pm 0.35$ \\
Borderzone 2 & $0.946 \pm 0.35$ \\
Infarct & $9.46 \pm 3.5$
\end{tabular}

was calculated according to the following equation (Sunagawa et al., 1983):

$\mathrm{SV}=\frac{\mathrm{LVV}_{\mathrm{ED}}-V_{0}}{1+E_{\mathrm{A}} / E_{\mathrm{ES}}}$

where $\mathrm{SV}$ is the stroke volume, $\mathrm{LVV}_{\mathrm{ED}}$ is the $\mathrm{LV}$ volume at the end diastole, $V_{O}$ is the volume axis intercept of end-systolic elastance, and $E_{\mathrm{ES}}$ is the slope of end-systolic elastance shown in Eq. 1. The arterial elastance, $E_{\mathrm{A}}$, was fixed for each sheep and was experimentally determined as the ratio of end-systolic pressure to SV (Sunagawa et al., 1985).

\section{STATISTICAL ANALYSIS}

All values are expressed as mean $\pm \mathrm{SD}$ and compared by repeated measures regression analysis using a mixed model to test for both fixed and random effects (Systat Software, Inc., Chicago, IL, USA). The effect of borderzone contractility was treated as a fixed effect. The enhanced borderzone contractility state was assumed to be a repeated event and individual animals treated as a random effect. Significance was set at $p$ less than 0.05 .

\section{RESULTS}

The principal finding of this study is that an increase in borderzone contractility has a substantial beneficial effect on LV pump function. Even partial increase in borderzone contractility was sufficient to drive much of the calculated increase in function.

The reliability of the FE model's predictions was tested with a mesh convergence study to find the minimum number of elements needed to produce accurate results within the fastest computation time. The mesh convergence study determined that 2496 elements is required and further mesh refinement only results in a $1 \%$ change in strain prediction.

\section{MIDWALL FIBER STRAIN AND STRESS BY REGIONS AT END SYSTOLE}

Figure 2 shows end-systolic myofiber strain (Figure 2A) and stress (Figure 2B) by regions (infarct, border zone 1, border zone 2, and remote) at POST MI, and after four different levels of BZ IMPROVEMENT (namely 25, 50, 75, and 100\%). Strain in the remote region was not changed after BZ-contractility improvement. Strain in BZ 1 was significantly reduced after $25 \%$ or more BZ IMPROVEMENT. Strain in BZ 2 was significantly reduced after $75 \%$ or more BZ improvement.

BZ IMPROVEMENT led to significantly higher midwall fiber stress at end systole in BZ 1. The end-systolic midwall fiber stress after 50\% BZ IMPROVEMENT, for example, was 16.7\% higher than that of the baseline. Interestingly, both the 50 and

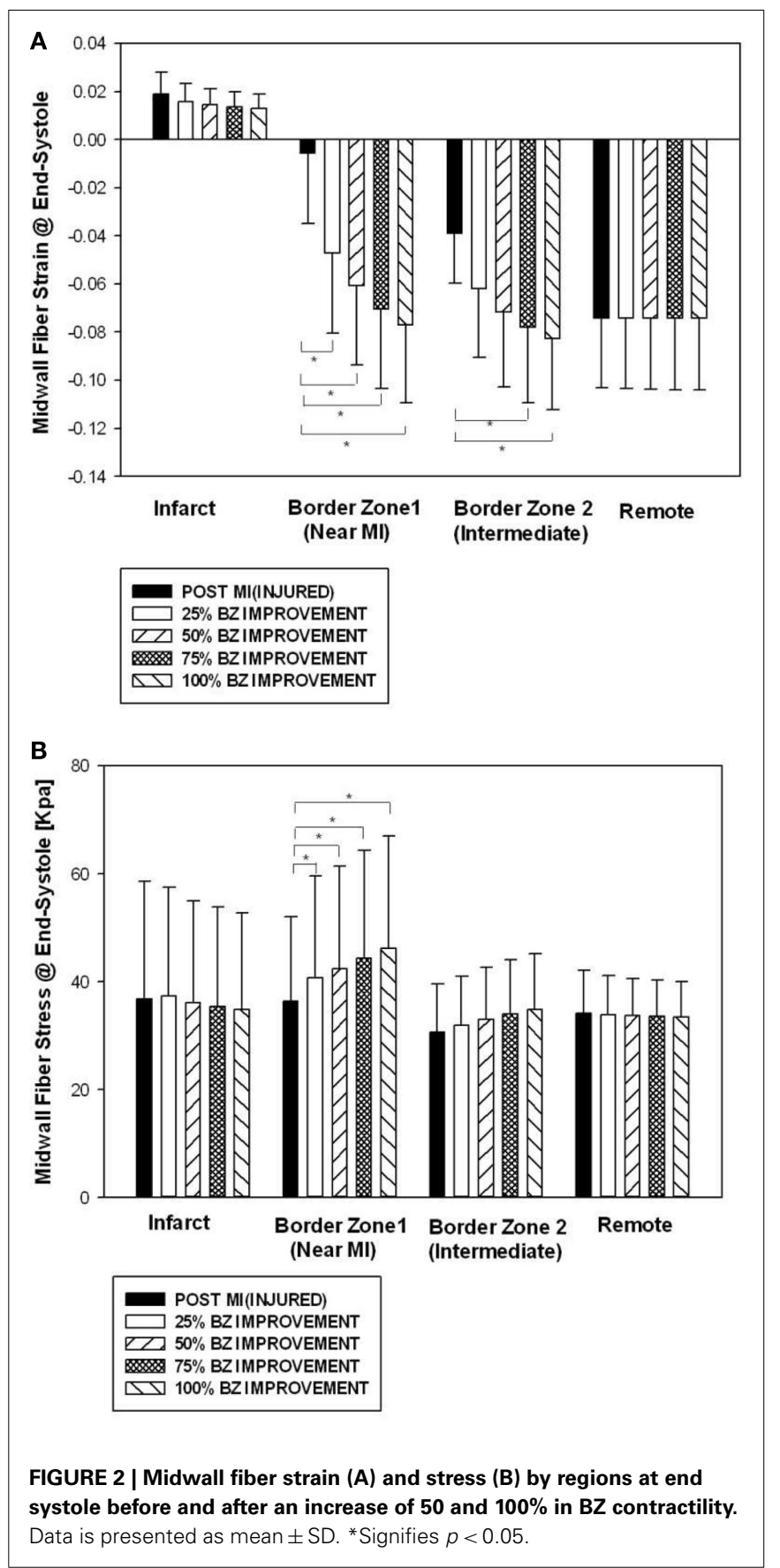

100\% BZ IMPROVEMENTs led fiber stress to be higher in BZ 1 than in the remote. In our FEM, the amount of fiber stress generated by myofiber contraction is determined by the contractility $T_{\max }$ and sarcomere length. The higher fiber stress in BZ 1 indicates higher end-systole sarcomere length in BZ 1 than the remote.

Figure 3 shows contours of myofiber stress at end systole at POST MI (Figure 3A) and after 50\% BZ IMPROVEMENT (Figure 3B) and 100\% BZ IMPROVEMENT (Figure 3C). These Figures reveal that there exists regional difference of the impact of borderzone contractility on myofiber stress. 


\section{BORDERZONE CONTRACTILITY AND LV PUMP FUNCTION}

Hundred percent BZ IMPROVEMENT shifted the end-systolic pressure volume relationship (ESPVR) to the left by $7.7 \mathrm{ml}$ at $110 \mathrm{mmHg}(p<0.001)$. Much of the beneficial effect was observed with 50\% BZ IMPROVEMENT which shifted the ESPVR to the left by $5.67 \mathrm{ml}$ at $110 \mathrm{mmHg}$ (Figure 4A).

Post-MI ejection fraction for the INJURED group is $17.1 \%$. After $100 \%$ BZ IMPROVEMENT ejection fraction is $23.4 \%$. An appreciable increase in ejection fraction was observed with only 50\% BZ IMPROVEMENT where ejection fraction was $21.6 \%$.
Hundred percent BZ IMPROVEMENT shifted the Starling curve upward with an appreciable $(36 \% ; p<0.001)$ increase in SV of $6.8 \mathrm{ml}$ at LVEDP $=20 \mathrm{mmHg}$. Similar to the effect on endsystolic elastance, much of the effect was observed with only $50 \%$ BZ IMPROVEMENT which increased SV $4.83 \mathrm{ml}(p<0.02)$ at $\mathrm{LVEDP}=20 \mathrm{mmHg}$ (Figure 4B). Figure 5 demonstrates the nonlinear nature of the relationship between improvement in BZ contractility and cardiac output. The SV improvement measured at $\mathrm{LVP}_{\mathrm{ED}}=20 \mathrm{mmHg}$ at 25 and 50\% BZ IMPROVEMENT was equivalent to 43 and $69 \%$ of the improvement seen at $100 \% \mathrm{BZ}$ IMPROVEMENT, respectively. The non-linear relationship was
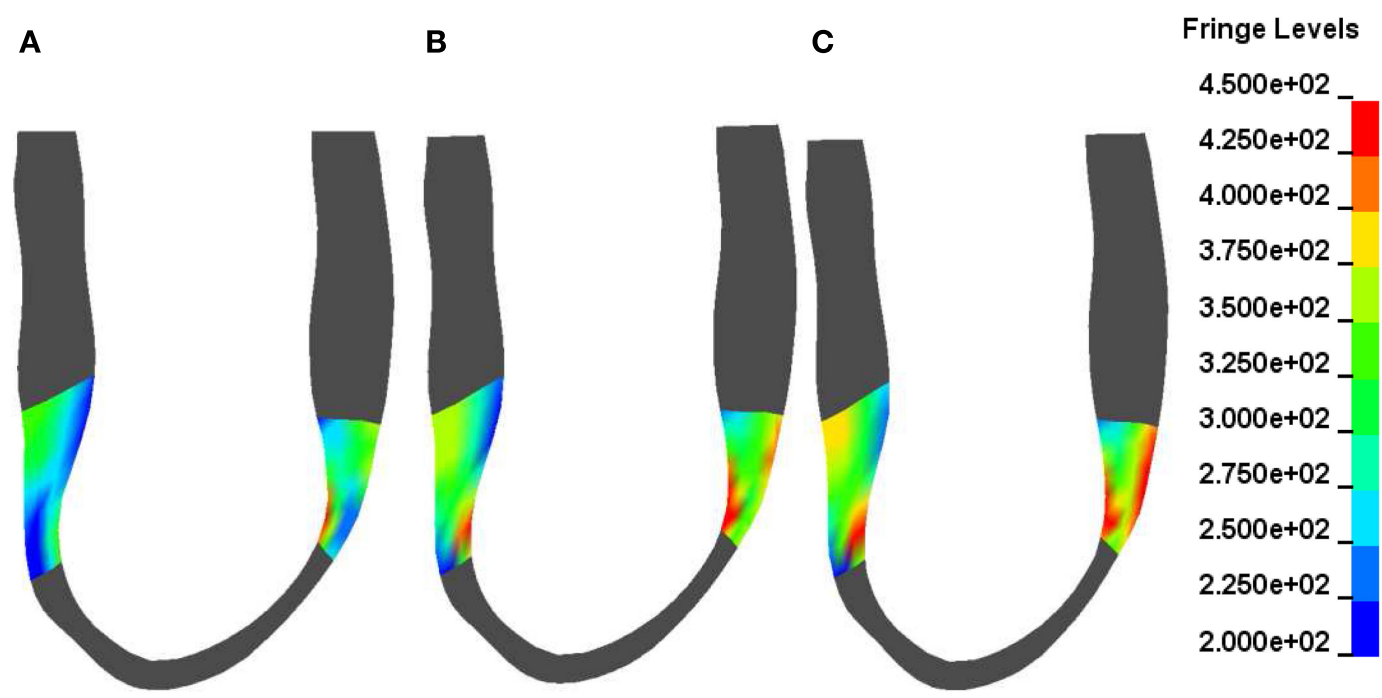

FIGURE 3 |Typical end-systole myofiber stress distribution in a sheep (corresponds to Figure 1) before (A) and after a $50 \%(B)$ and $100 \%$ (C) increase in BZ contractility. Fringe levels are in units of

$\mathrm{hPa}=0.1 \mathrm{kPa}=0.1 \mathrm{mN} / \mathrm{mm}^{2}$. Note the non-homogeneous BZ stress change after a 50 and $100 \%$ increase in BZ-contractility compared with the depressed BZ contractility.

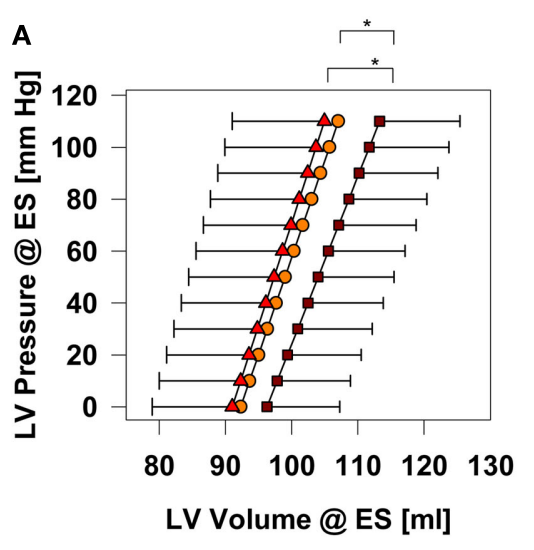

B
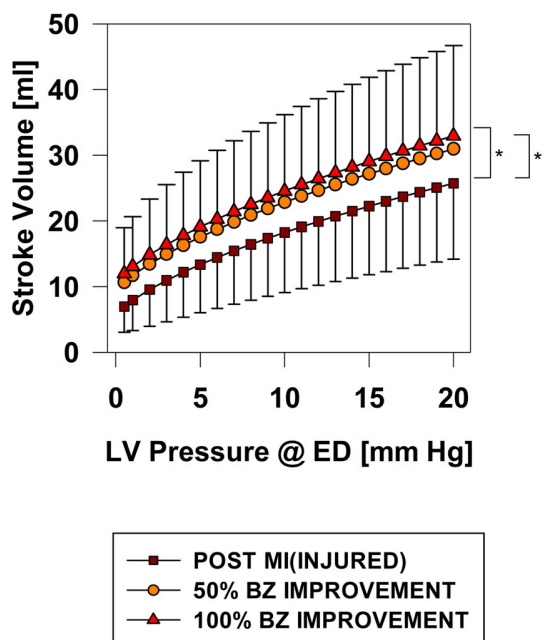

FIGURE 4 | End-systolic pressure volume (A) and Starling relationships (B) before and after an increase 50 and $\mathbf{1 0 0 \%}$ in BZ contractility. ${ }^{*}$ Signifies $p<0.001$. 


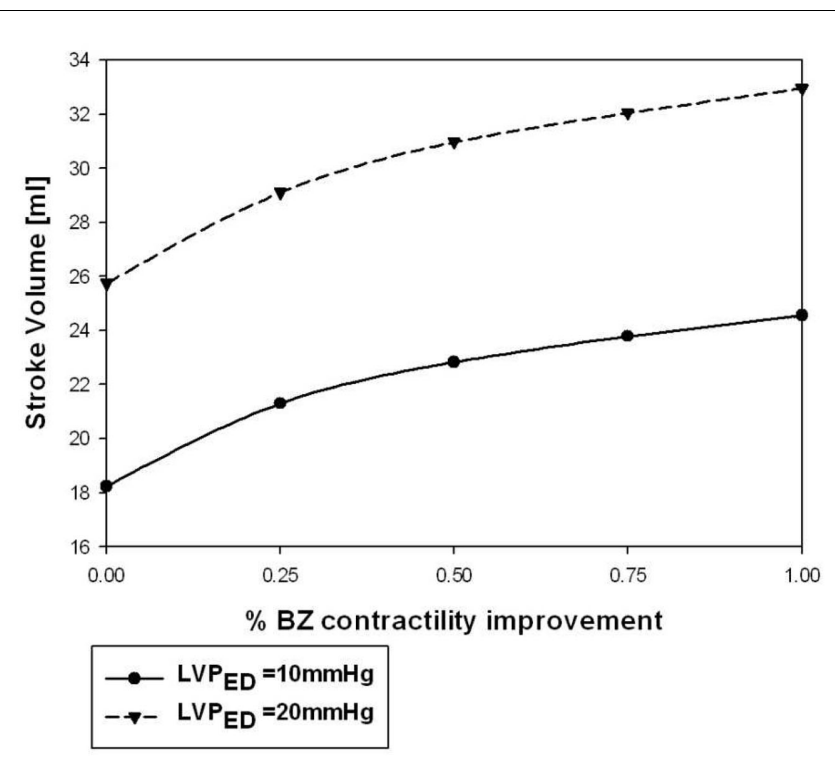

FIGURE 5 | Stroke volume at $L V P_{E D}=10$ and $20 \mathrm{mmHg}$ varied when BZ-contractility restored from 0 (INJURED) to $100 \%$ improvement.

apparently not sensitive to changes of LV end-diastolic pressure (Figure 5).

\section{DISCUSSION}

The principal finding of this study is that normalization of borderzone contractility, evenly partially, can significantly improve LV pump function.

\section{THE UTILITY OF VIRTUAL EXPERIMENTS}

The current study demonstrates how FE simulations can be used to predict the mechanical behavior of the heart under different pathophysiologic conditions. For example, the current study suggests that even partial recovery of BZ function can substantially improve overall LV function. FE simulations, however, do not obviate the need for subsequent animal and/or human experiments designed to confirm the computer based results since there are acute and chronic compensatory mechanisms that the models do not take into consideration. However, an investigator using FE simulations can rapidly test a large number of potential interventions/therapies. In fact, it may be possible to use computer based optimization algorithms to search for the optimal interventions (Stander and Craig, 2002) such as the greatest LV wall stress reduction or the largest increase in cardiac output. The investigator would then conduct animal and human experiments specifically targeted to test the findings of FE simulations. This approach has the potential to be vastly more efficient and fewer animals and human subjects would be needed to determine the effect of a specific mechanical intervention.

\section{IMPACT OF BZ-CONTRACTILITY IMPROVEMENT ON STRAIN AND STRESS}

It has been previously reported that myocyte stretch during contraction causes injury in skeletal muscles (Proske and Morgan,
2001). This mechanism could also play a role in the dysfunction of borderzone myocytes. At the baseline (Figure 2A), the average strain of BZ 1 is very low $(-0.005 \pm 0.03$, Mean \pm SD) and some myocytes in BZ 1 could be stretched during contraction. Interestingly, when the borderzone contractility is increased by $25 \%$ toward the remote value, the systolic contractive strain in BZ 1 increased $(-0.05 \pm 0.04)$. Further borderzone contractility improvement to $50 \%$ toward the remote value leads to a $\mathrm{BZ} 1$ strain of $-0.06 \pm 0.03$. Such a finding suggests that borderzone contractility improvement could potentially protect borderzone myocytes from systolic stretching thus prevent further functional reduction.

There exists a regional difference of the effect of BZcontractility improvement on wall stress. As seen in Figure 2B, the wall stress in the remote region and BZ 2 (the borderzone far from the infarct region) was not significantly altered after contractility improvement. BZ 1 wall stress, however, was increased after contractility improvement. The mechanical tethering effect of the infarct region is probably partly responsible for the difference between BZ 1 and BZ 2. The indication of such regional wall stress alteration on long term LV pump function is unclear immediately and needs to be investigated through animal model studies.

\section{NON-LINEAR RELATIONSHIP BETWEEN THE IMPROVEMENTS OF LV PUMP FUNCTION AND BZ CONTRACTILITY}

Figure 5 showed that the percentage of LV pump function improvement is non-linearly related to the percentage increasement of BZ contractility. Such a non-linear effect is partly due to the fact that the contractility improvement is restricted to the BZs while the remote region contractility is intact. The non-linearity is also partly due the non-linear relationship between active myocyte tension development and sarcomere length. Fortunately, a larger percentage of LV pump function improvement occurs at the lower BZ-contractility improvement region. As seen in Figure 5, a 25\% improvement of BZ contractility is able to produce $43 \%$ of the LV pump function improvement seen in the $100 \%$ contractility improvement case. Such a non-linear performance makes the approach of BZ-contractility enhancement very attractive.

\section{Potential borderzone contractility improvement therapies}

Surgical ventricular remodeling. As above, borderzone dysfunction is probably initiated by stretch during contraction in the borderzone myocardium. Since patch aneurysmorrhaphy reduces stress in the borderzone surrounding an anteroapical myocardial infarction, it was hoped that borderzone contractility would improve after surgical ventricular remodeling (Sun et al., 2010). However, we recently measured borderzone contractility after the Dor procedure in sheep and found that it was not improved (Sun et al., 2010). The amount of stress reduction was either not sufficient or the myocardium was so damaged that it could not recover.

Passive constraint. Placement of an inextensible mesh around the apex of the heart preserves the geometry and function of the LV after acute anteroapical infarction (Kelley et al., 1999). In addition, application of the Acorn CorCap Cardiac Support Device after MI reduces the amount of akinesis (Pilla et al., 2002) although 
significant remodeling still occurs. The effect of an inextensible on borderzone contractility would be of significant interest.

Cell transplantation. Jameel et al. (2010) injected bone marrow derived progenitor cells into the borderzone in pigs immediately after anterior MI. There were no surviving transplanted cells 4 months after transplantation. However, a significant improvement in ejection fraction that was seen early after transplantation persisted to 4 months. Furthermore, the PCr/ATP ratio was increased as much as $15 \%$ in the borderzone endocardium (Jameel et al., 2010). The cause of this beneficial effect is unknown but could be partly caused by improvement in borderzone contractility.

Matrix metalloproteinase inhibition therapy. Extracellular matrix degradation by matrix metalloproteinase (MMPs) is a known factor leading to the LV remodeling after myocardial infarction. MMP inhibition has been shown to prevent LV dilatation in mice and rabbits (Rohde et al., 1999; Lindsey et al., 2002). We recently tested the hypothesis that MMP inhibition could slow down the borderzone dysfunction after MI in a sheep model. Preliminary results indicate that MMP inhibition with doxycycline

\section{REFERENCES}

Guccione, J. M., Moonly, S. M., Moustakidis, P., Costa, K. D., Moulton, M. J., Ratcliffe, M. B., and Pasque, M. K. (2001). Mechanism underlying mechanical dysfunction in the border zone of left ventricular aneurysm: a finite element model study. Ann. Thorac. Surg. 71, 654-662.

Homans, D. C., Asinger, R., Elsperger, K. J., Erlien, D., Sublett, E., Mikell, F., and Bache, R. J. (1985). Regional function and perfusion at the lateral border of ischemic myocardium. Circulation 71, 1038-1047.

Jackson, B. M., Gorman, J. H., Moainie, S. L., Guy, T. S., Narula, N., Narula, J., John-Sutton, M. G., Edmunds, L. H. Jr., and Gorman, R. C. (2002). Extension of borderzone myocardium in postinfarction dilated cardiomyopathy. J. Am. Coll. Cardiol. 40, 1160-1167; discussion 1168-1171.

Jameel, M. N., Li, Q., Mansoor, A., Qiang, X., Sarver, A., Wang, X., Swingen, C., and Zhang, J. (2010). Long-term functional improvement and gene expression changes after bone marrow-derived multipotent progenitor cell transplantation in myocardial infarction. Am. J. Physiol. Heart Circ. Physiol. 298, H1348H1356.

Kelley, S. T., Malekan, R., Gorman, J. H. III, Jackson, B. M., Gorman, R. C., Suzuki, Y., Plappert, T., Bogen, D. K., Sutton, M. G., and Edmunds, L. H. Jr. (1999). Restraining infarct expansion preserves left ventricular geometry and function after acute anteroapical infarction. Circulation 99, 135-142. Soleimani, M., Wu, Y., Baker, A. J., and Ratcliffe, M. B. (2011). Doxycyline prevents borderzone contractility reduction after myocardial infarction in sheep. J. Am. Coll. Surg. 213, S33.

Kleinbaum, D., Kupper, L., and Muller, K. (1988). Applied Regression AnalyBoston: PWS-Kent. T. M., Power, T. P., Petruolo, S., and Reichek, N. (1996). Remote noninfarcted region dysfunction soon after first anterior myocardial infarction. A magnetic resonance tagging study. Circulation 94, 660-666.

Lindsey, M. L., Gannon, J., Aikawa, M., Schoen, F. J., Rabkin, E., LoprestiMorrow, L., Crawford, J., Black, S., Libby, P., Mitchell, P. G., and Lee, R. T. (2002). Selective matrix metalloproteinase inhibition reduces left ventricular remodeling but does not inhibit angiogenesis after myocardial infarction. Circulation 105, 753-758.

Pilla, J. J., Blom, A. S., Brockman, D. J., Bowen, F., Yuan, Q., Giammarco, J., Ferrari, V. A., Gorman, J. H. III, Gorman, R. C., and Acker, M. A. (2002). Ventricular constraint using the acorn cardiac support device reduces myocardial akinetic area in an ovine model of acute infarction. Circulation 106, I207-I211.

Proske, U., and Morgan, D. L. (2001). Muscle damage from eccentric
Khazalpour, M., Shimkunas, R., sis and Other Multivariable Methods.

Kramer, C. M., Rogers, W. J., Theobald,

prevents borderzone myofiber contractility reduction after MI (Khazalpour et al., 2011).

\section{LIMITATIONS}

There are a few limitations in this study. First, these FE models have assumed that the contractility is homogeneous within the pre-defined BZ, remote, and infarct regions. As a result, contractility changed abruptly at the infarction-BZ and the BZ-remote boundaries. The second limitation is the absence of the RV in the FE model and the future studies will need to include the RV in the model.

\section{CONCLUSION}

Even partial improvement of borderzone contractility has a substantial beneficial effect on LV pump function. Therapies specifically designed to improve borderzone contractility should be developed.

\section{ACKNOWLEDGMENTS}

This study was supported by NIH grant R01-HL-77921 (Dr. Guccione), and R01-HL-63348 (Dr. Ratcliffe). This support is gratefully acknowledged.

exercise, mechanism, mechanical signs, adaptation and clinical applications. J. Physiol. 537, 333-345.

Rohde, L. E., Ducharme, A., Arroyo, L. H., Aikawa, M., Sukhova, G. H., Lopez-Anaya, A., McClure, K. F., Mitchell, P. G., Libby, P., and Lee, R. T. (1999). Matrix metalloproteinase inhibition attenuates early left ventricular enlargement after experimental myocardial infarction in mice. Circulation 99 3063-3070.

Stander, N., and Craig, K. J. (2002). On the robustness of a simple domain reduction scheme for simulation based optimization. Eng. Comput. 19, 431-450.

Sun, K., Stander, N., Jhun, C.-S., Zhang, Z., Suzuki, T., Saeed, M., Wallace, A., Tseng, E., Baker, A., Saloner, D., Einstein, D., Ratcliffe, M., and Guccione, J. (2009). A computationally efficient formal optimization of regional myocardial contractility in a sheep with left ventricular aneurysm. J. Biomech. Eng. 131, 111001.

Sun, K., Zhang, Z., Suzuki, T., Wenk, J. Stander, N., Einstein, D., Saloner, D. Wallace, A., Guccione, J., and Ratcliffe, M. (2010). Dor procedure for dyskinetic anteroapical myocardial infarction fails to improve contractility in the borderzone. J. Thorac. Cardiovasc. Surg. 140, 233-239.

Sunagawa, K., Maughan, W. L., Burkhoff, D., and Sagawa, K. (1983). Left ventricular interaction with arterial load studied in isolated canine ventricle. Am. J. Physiol. Heart Circ. Physiol. 245, H773-H780.
Sunagawa, K., Maughan, W. L., and Sagawa, K. (1985). Optimal arterial resistance for the maximal stroke work studied in isolated canine left ventricle. Circ. Res. 56, 586-595.

Walker, J. C., Ratcliffe, M. B., Zhang, P., Wallace, A. W., Fata, B., Hsu, E. W., Saloner, D., and Guccione, J. M. (2005). MRI-based finite-element analysis of left ventricular aneurysm. Am. J. Physiol. Heart Circ. Physiol. 289, H692-H700.

Conflict of Interest Statement: The authors declare that the research was conducted in the absence of any commercial or financial relationships that could be construed as a potential conflict of interest.

Received: 14 December 2011; accepted: 22 March 2012; published online: 09 April 2012.

Citation: Zhang Z, Sun K, Saloner DA, Wallace $A W$, Ge L, Baker AJ, Guccione JM and Ratcliffe MB (2012) The benefit of enhanced contractility in the infarct borderzone: a virtual experiment. Front. Physio. 3:86. doi: 10.3389/fphys.2012.00086

This article was submitted to Frontiers in Computational Physiology and Medicine, a specialty of Frontiers in Physiology. Copyright (C) 2012 Zhang, Sun, Saloner, Wallace, Ge, Baker, Guccione and Ratcliffe. This is an open-access article distributed under the terms of the Creative Commons Attribution Non Commercial License, which permits noncommercial use, distribution, and reproduction in other forums, provided the original authors and source are credited. 